\title{
Review
}

\section{Bacterial bile salt hydrolase: an intestinal microbiome target for enhanced animal health}

\author{
Wenjing Geng and Jun Lin* \\ Department of Animal Science, The University of Tennessee, Tennessee, USA
}

Received 8 April 2016; Accepted 12 August 2016

\begin{abstract}
To effectively mitigate antimicrobial resistance in the agricultural ecosystem, there is an increasing pressure to reduce and eliminate the use of in-feed antibiotics for growth promotion and disease prevention in food animals. However, limiting antibiotic use could compromise animal production efficiency and health. Thus, there is an urgent need to develop effective alternatives to antibiotic growth promoters (AGPs). Increasing evidence has shown that the growth-promoting effect of AGPs was highly correlated with the reduced activity of bile salt hydrolase $(\mathrm{BSH})$, an intestinal bacterial enzyme that has a negative impact on host fat digestion and energy harvest; consistent with this finding, the population of Lactobacillus species, the major intestinal BSH-producer, was significantly reduced in response to AGP use. Thus, BSH is a key mechanistic microbiome target for developing novel alternatives to AGPs. Despite recent significant progress in the characterization of diverse $\mathrm{BSH}$ enzymes, research on $\mathrm{BSH}$ is still in its infancy. This review is focused on the function of BSH and its significant impacts on host physiology in human beings, laboratory animals and food animals. The gaps in BSH-based translational microbiome research for enhanced animal health are also identified and discussed.
\end{abstract}

Keywords: bile salts, bile salt hydrolase, lipid metabolism, antibiotic growth promoters, non-antibiotic feed additives, intestinal microbiome.

\section{Introduction}

Antibiotic use clearly serves as a selective driving force to enrich antimicrobial resistance (AMR) genes and promote the emergence of antibiotic-resistant bacterial pathogens (Davies, 2014). Thus, reducing or eliminating the use of in-feed antibiotics in healthy animals has been a worldwide trend to effectively mitigate AMR and protect food safety. US Food and Drug Administration recently implemented a new policy to recommend a voluntary withdrawal of medically important antibiotic from routine animal production practices by December 2016. Therefore, there is an urgent need to develop effective strategies to maintain animal productivity and health without relying on infeed antibiotics.

Food animal producers have manipulated intestinal microbiota for more than 60 years to increase feed efficiency and

*Corresponding author. E-mail: jlin6@utk.edu body weight gain through the routine use of low-dose antibiotics as feed additives, called antibiotic growth promoters (AGPs). With the aid of culture-independent molecular approaches, investigations of the effect of AGPs on intestinal microbiota have been initiated in different food animals, including poultry and swine (Lin, 2014). These microbiome studies have shed light on the mechanism of mode of action of AGPs and on the development of novel alternatives to AGPs. Specifically, data indicate that the body weight gain in food animals is inversely related to the activity of bile salt hydrolase $(\mathrm{BSH})$ as well as the abundance of potent BSH-producing bacteria in the intestine (Lin, 2014). Because the BSH enzymes produced by intestinal bacteria catalyze deconjugation of conjugated bile acids, an essential gateway reaction in the metabolism of bile acids which play an important role in host fat metabolism, energy harvest and body weight gain (Begley et al., 2006; Joyce et al., 2014b), we propose that BSH is a key mechanistic microbiome target for developing novel alternatives to AGPs, such as $\mathrm{BSH}$ inhibitors for enhanced animal production and health. This 
article reviews recent progress on $\mathrm{BSH}$ research, with emphasis on $\mathrm{BSH}$ functions and its impact on host physiology.

\section{Bile acids}

Primary bile acids are de novo synthesized from cholesterol in the liver and are conjugated to either glycine or taurine to form conjugated bile acids (Appleby and Walters, 2014; Schaap et al., 2014; Camilleri and Gores, 2015). The amphipathic characteristic of conjugated bile acid helps dietary lipids or fat-soluble vitamins form micelles, which facilitate their metabolism by pancreatic enzymes prior to their absorption (de Aguiar Vallim et al., 2013). Thus, conjugated bile acids are more efficient than unconjugated bile acids for emulsification and digestion of dietary lipids or lipid soluble nutrients (Hofmann and Mysels, 1992; Ridlon et al., 2006). Following synthesis, bile salts are stored and concentrated in the gallbladder. Upon food consumption, chyme from partly digested food is expelled from stomach into the duodenum, acids and partially digested fat stimulate the secretion of secretin and cholecystokinin (CCK) (Begley et al., 2005). Subsequently, CCK stimulates the contraction of the gallbladder, and leads to the release of bile salts from the gallbladder into the small intestine for lipid digestion (Johnson, 1998). In animals without a gallbladder, such as horses and rats, bile salts continuously flow directly from the liver to the duodenum via the bile duct.

After reaching the ileum, bile salts are taken up into enterocytes via efficient membrane transporters, further absorbed into the portal vein to get back to the liver and finally re-secreted into bile; this process is called enterohepatic circulation (Vlahcevic et al., 1996; Roberts et al., 2002; Begley et al., 2006; Ridlon et al., 2006; Russell, 2009). In human beings, approximately 400-800 mg of bile salts daily are subjected to microbial transformations in the intestine (Vlahcevic et al., 1996). Among various bile salt transformations, deconjugation of conjugated bile salts is the gateway reaction for bile alteration and is a prerequisite for all sterol transformation (Batta et al., 1990; Kim and Lee, 2005). Notably, in addition to a direct digestive role in the emulsification of dietary fats in the intestine, bile acids can act as signaling molecules to affect energy metabolism, bile acids enterohepatic circulation, host cholesterol level, and triglyceride and glucose homeostasis (Joyce et al., 2014b). In particular, unconjugated bile acids have been shown to specifically interact with orphan nuclear hormone receptors such as farnesoid X receptor (FXR) and G-protein-coupled receptor TGR5 (Gupta et al., 2001; Qiao et al., 2003; Houten et al., 2006; Inagaki et al., 2006; Evans et al., 2009).

\section{Bile salt hydrolase}

The BSH enzyme produced by intestinal bacteria catalyzes deconjugation of conjugated bile acids by hydrolyzing the amide bond and producing free amino acids and unconjugated bile acids; this is an essential gateway reaction in the metabolism of bile acids in the small intestine (Begley et al., 2006). BSH enzyme belongs to the choloylglycine hydrolase (EC 3.5.1.24) family. Phylogenetic analysis indicated that $\mathrm{BSH}$ was derived from the wider Ntn_CGH-like family of proteins, specifically penicillin V acylase (Kumar et al., 2006; Jones et al., 2008).

$\mathrm{BSH}$ enzymes from various sources differ in activity, substrate specificity, and optimal temperature and $\mathrm{pH}$ for enzymatic activity (Begley et al., 2006). Molecular weights of the BSH subunit range from 28 to $50 \mathrm{kDa}$, and optimal $\mathrm{pH}$ for $\mathrm{BSH}$ activity is slightly acidic, ranging from 3.5 to 6 . Most identified $\mathrm{BSH}$ enzymes still display activity at temperatures up to $60^{\circ} \mathrm{C}$. Many identified BSH enzymes have a narrow substrate spectrum and display much higher activity in hydrolyzing glycineconjugated bile salts than taurine-conjugated bile salts (Coleman and Hudson, 1995; Smet et al., 1995; Tanaka et al., 2000; Kim et al., 2004; Liong and Shah, 2005; Pavlović et al., 2012). However, some BSH enzymes show a preference for taurine-conjugated bile salts, such as two BSH enzymes in Lactobacillus jonsonii PF01 (Chae et al., 2013) and the BSH enzymes from five lactobacilli strains (Jiang et al., 2010). Recently, a potent BSH enzyme was identified and characterized from a chicken Lactobacillus salivarius strain; this BSH displayed potent hydrolysis activity towards both glycol-conjugated and taurine-conjugated bile salts (Wang et al., 2012). It has been proposed that $\mathrm{BSH}$ enzymes recognize conjugated bile acids on both amino acid moieties and the cholate steroid nucleus (Begley et al., 2006). Not surprisingly, substrate preferences of BSH may differ under different $\mathrm{pH}$, likely due to pH-mediated structural changes (Corzo and Gilliland, 1999).

To date, structural basis of BSH function is still largely unknown. Crystal structures of the BSH enzymes from only three specific species, Bifidobacterium longum, Clostridium perfrigens, and L. salivarius have been reported (Rossocha et al., 2005; Kumar et al., 2006; Xu et al., 2016). The $1.90 \AA$ crystal structure of the L. salivarius BSH was recently determined by molecular replacement using the starting model of $C$. perfringens $\mathrm{BSH}(\mathrm{Xu}$ et al., 2016). Comparative structural analysis of the $L$. salivarius $\mathrm{BSH}$ also identified potential residues contributing to catalysis and substrate specificity. Together, unlike the binding pocket in other BSHs such as the C. perfrigens BSH that shows an open entrance with shallow bottom, a panel of unique residues in the L. salivarius BSH make this BSH display narrow entrance of the binding pocket and the increased inner capacity of the binding pocket, which may enable substrates to sit deeply in the pocket with different conformation and lead to the broad spectrum of specificity (Wang et al., 2012; Xu et al., 2016). Previous comparative genomics and structural studies have identified some conserved, catalytically important residues in the active site of BSH (Cys2, Arg 16, Asp19, Asn79, Asn171, and Arg224); however, this conclusion was primarily based on the comparison of $\mathrm{BSH}$ structure with penicillin $\mathrm{V}$ acylase (Begley et al., 2006; Kumar et al., 2006; Wang et al., 2012). To date, Cys2 is the only residue that has been subjected to sitedirected mutagenesis and validated for its essential role in BSH activity (Kumar et al., 2006). Therefore, future in-depth structural analysis of the unique $L$. salivarius BSH (e.g. in complex with specific substrate) in conjunction with comprehensive amino acid substitution mutagenesis would help to discover 
residues critical in catalysis and understand why this $\mathrm{BSH}$ displayed potent catalytic activity toward a broad spectrum of substrates including both glycol-conjugated and taurine-conjugated bile salts.

\section{BSH-producing bacteria in the intestine}

BSH enzymes have been identified in diverse bacterial species from different sources (Summarized in Table 1). Among the $\mathrm{BSH}$-producing organisms, most of them are Gram-positive bacteria, except two from the Gram-negative genus, Bacteroides (Stellwag and Hylemon, 1976; Masuda, 1981; Lambert et al., 2008). Jones et al. (2008) performed a functional and comparative metagenomic analysis of BSH activity in the human intestinal microbiome and showed a high level of redundancy of $\mathrm{BSH}$ distribution in the human intestine ecosystem; most BSH activity was distributed in all major phyla within intestinal microbiota (primarily Firmicutes, followed by Bacterioidetes and Actinobacteria) and across two domains of life (Bacteria and Archaea in the intestine) (Jones et al., 2008).

$\mathrm{BSH}$ genes are particularly abundant in lactic acid fermenting probiotics, such as lactobacilli and bifidobacteria, which are the species most commonly used as probiotics due to their healthpromoting activities (Reviewed by Begley et al., 2006). As shown in Table 1, BSH activity and corresponding enzymes have been identified primarily in lactic acid bacteria isolated from the gastrointestinal tract, which include but are not limited to L. salivarius, Lactobacillus acidophilus, Lactobacillus johnsonii, Lactobacillus plantarum, Bifidobacterium longum, Bifidobacterium bifidum, Bifidobacterium adolescentis, and Bifidobacterium animalis. BSH genes are either located in the chromosome or in mobile element, such as the megaplasmid identified in L. salivarius UCC118 (Claesson et al., 2006). It is not unusual that multiple BSH homologs, which are not identical, could be present in a single intestinal bacterial strain (Begley et al., 2006; Wang et al., 2012). It has been speculated that $\mathrm{BSH}$ genes may be acquired horizontally among intestinal microorganisms (Begley et al., 2006). However, there is no compelling evidence demonstrating horizontal transfer of BSH genes in intestinal microorganisms.

Jones et al. (2008) also have determined that active BSH enzymes are restricted to intestinal microorganisms, suggesting that BSH activity plays a role in in vivo adaptation of intestinal microorganisms in the gastrointestinal environment and in the mutualism between intestinal microbiota and animal hosts (Jones et al., 2008). Physiological advantages of BSH for bacterial producers themselves are still not well understood. One popular opinion is that $\mathrm{BSH}$ activity contributes to the resistance of commensal bacteria towards bile salts, a natural antimicrobial present in the intestine (Begley et al., 2006). For example, it has been demonstrated that BSH activity plays an important role in the bile resistance and intestinal colonization of Listeria innocua in a mouse model (Jones et al., 2008). However, the unconjugated bile salts resulting from BSH hydrolysis could still display antimicrobial activity; thus, there are contradictory findings about contribution of $\mathrm{BSH}$ activity to bile tolerance in intestinal probiotic bacteria (Begley et al., 2006). At present, there is no convincing in vivo evidence demonstrating that BSH enzyme contributes to bile resistance in probiotic bacteria, such as lactobacilli. Fang et al. (2009) demonstrated that production of BSH does not determine the bile resistance level in L. salivarius, the dominant Lactobacillus species present in animal intestine (Fang et al., 2009). In addition to this popular hypothesis, there are some other opinions about the roles of BSH in bacterial physiology based on some evidence in certain commensal bacteria. For example, it has been proposed that hydrolysis of conjugated bile acids by BSH can provide cellular carbon, nitrogen, sulfur as well as energy source for some bacteria species (Vlahcevic et al., 1996; Tanaka et al., 2000; Ridlon et al., 2006). BSH may also trigger the influx of cholesterol or bile into bacterial cells and increase membrane electrochemical characteristics, which may facilitate some microorganisms to inhabit in the gastrointestinal epithelium in the host via immune evasion (Jones et al., 2008; Mukherji and Prabhune, 2015).

\section{The impact of bacterial BSH activity on host physiology}

Despite the lack of understanding of the benefits of $\mathrm{BSH}$ for $\mathrm{BSH}$-producing bacteria, it has been well recognized that intestinal BSH plays an important role in host lipid metabolism, dietary energy harvest and body weight gain because BSH catalyzes the gateway reaction in the metabolism of bile acids in the intestine (Begley et al., 2006; Jones et al., 2008; Joyce et al., 2014b). To date, functional research on the relationship between bacterial $\mathrm{BSH}$ and host physiology/health have been primarily focused on human probiotics using laboratory animal model systems. There are very limited efforts to determine the impact of intestinal bacterial BSH activity on growth and health in food animals (Feighner and Dashkevicz, 1988; Knarreborg et al., 2004; Guban et al., 2006; Lin, 2011). The following paragraphs summarize findings from laboratory animal studies and human trials, which shed light on future directions for food animal health research.

\section{Host lipid metabolism, cholesterol, and body weight}

As children and adults are increasingly becoming overweight and obese, obesity-associated diseases will increase (Kahn et al., 2006; Van Gaal et al., 2006). Recent studies have indicated that intestinal microbiota are implicated in obesity in people (Tremaroli and Bäckhed, 2012); however, key microbial functions influencing host energy harvest remain to be clearly elucidated. The BSH enzyme has been increasingly recognized as a critical intestinal microbiome target for developing intervention strategy to control obesity.

Given that the bile acids have dual digestive and signaling roles in the host, intestinal BSH plays an important role in host metabolism and energy harvest; $\mathrm{BSH}$ activity has significant impacts on host physiology by disturbing conjugated bile acid-mediated fat metabolism and endocrine functions (Begley et al., 2006; Patel et al., 2010; Jones et al., 2014; Joyce et al., 
Table 1. The BSH enzymes identified in bacteria from various sources

\begin{tabular}{|c|c|c|c|c|c|}
\hline Source & Host strain & Molecular mass (kDa) & pH optimum & Temperature optimum $\left({ }^{\circ} \mathrm{C}\right)$ & Reference \\
\hline \multicolumn{6}{|l|}{ Human intestine } \\
\hline & Bacteroides fragilis ATCC 25285 & 32.5 & $4.2-4.5$ & ND & Stellwag and Hylemon (1976) \\
\hline & Bacteroides fragilis NCTC 9343 & ND & $5.0-6.0$ & ND & Aries and Hill (1970) \\
\hline & Bacteroides fragilis 2536 & ND & $4.5-5.0$ & ND & Masuda (1981) \\
\hline & Bacteroides vulgatus I-1 & ND & $4.5-5.0$ & ND & Masuda (1981) \\
\hline & Bacteroides vulgatus VI 31 & 36 & $5.6-6.4$ & ND & Kawamoto et al. (1989) \\
\hline & Bifidobacterium longum BB536 & 40 & $5.5-6.5$ & $35-40$ & Grill et al. (1995) \\
\hline & Bifidobacterium longum SBT2928 & 35 & $5.0-7.0$ & $40-45$ & Tanaka et al. (2000) \\
\hline & Bifidobacterium bifidum ATCC 11863 & 35 & ND & ND & Kim et al. (2004) \\
\hline & Bifidobacterium adolescentis ATCC 15705 & 35 & ND & ND & Kim et al. (2005) \\
\hline & Clostridium perfringens ATCC 19574 & ND & $5.6-5.8$ & ND & Nair et al. (1967) \\
\hline & Clostridium perfringens PB $6 \mathrm{~K}$ & ND & $4.5-5.0$ & ND & Masuda (1981) \\
\hline & Clostridium sordellii 4709 & ND & $4.5-5.0$ & ND & Masuda (1981) \\
\hline & Lactobacillus acidophilus L1 & $126^{\mathrm{a}}$ & $3.5-5.5$ & ND & Corzo and Gilliland (1999) \\
\hline & Lactobacillus acidophilus $\mathrm{O} 16$ & $126^{\mathrm{a}}$ & $3.5-6.0$ & ND & Corzo and Gilliland (1999) \\
\hline & Lactobacillus acidophilus NCFM & ND & ND & ND & McAuliffe et al. (2005) \\
\hline & Lactobacillus sp. strain 100-12 & ND & ND & ND & Lundeen and Savage (1990) \\
\hline & Listeria monocytogenes & ND & ND & ND & Dussurget et al. (2002) \\
\hline \multicolumn{6}{|l|}{ Murine intestine } \\
\hline & Lactobacillus sp. strain 100-100 & 42 & $3.8-4.5$ & ND & Lundeen and Savage (1990) \\
\hline & Lactobacillus sp. strain 100-16 & ND & ND & ND & Lundeen and Savage (1990) \\
\hline & Lactobacillus sp. strain RI & ND & ND & ND & Lundeen and Savage (1990) \\
\hline \multicolumn{6}{|l|}{ Pig intestine } \\
\hline & Lactobacillus acidophilus ATCC 43121 & $126^{\mathrm{a}}$ & $3.5-5.5$ & ND & Corzo and Gilliland (1999) \\
\hline & Lactobacillus acidophilus PF01 & 35 & 6 & 40 & Oh et al. (2008) \\
\hline & Lactobacillus johnsonii PF01 & $36 \& 37$ & 5.0 & $55(\mathrm{BSH} \mathrm{A}) \& 70(\mathrm{BSH} \mathrm{C})$ & Chae et al. (2013) \\
\hline & Lactobacillus sp. strain 100-33 & ND & ND & ND & Lundeen and Savage (1990) \\
\hline chicken intestıne & Lactobacillus salivarius NRRL B-30514 & 37 & $5.0-6.0$ & $35-55$ & Wang et al. (2012) \\
\hline \multicolumn{6}{|c|}{ - } \\
\hline Fermented milk & Bifidobacteriu. animalis DN 173010 & ND & ND & ND & Lepercq et al. (2004) \\
\hline Springs & Brevibacullus sp. & 28 & 9 & 60 & Sridevi et al. (2009) \\
\hline Fermented milk & Clostridium perfringens MCV 815 & 56.0 & $5.8-6.4$ & ND & $\begin{array}{l}\text { Gopal-Srivastava and Hylemon } \\
\text { (1988) }\end{array}$ \\
\hline Fermented finger millet & Pediococcus pentosaceus KID7 & ND & ND & ND & Damodharan et al. (2015) \\
\hline Fermented milk & Lactobacillus acidophilus sp. & ND & ND & ND & Pinto et al. (2006) \\
\hline Parakeet & Lactobacillus salivarius LMG 14476 & $140-142^{\mathrm{a}}$ & $5.5-7.0$ & ND & Bi et al. (2013), Li et al. (2006) \\
\hline Raw milk & Lactobacillus plantarum & ND & ND & ND & Sieladie et al. (2011) \\
\hline Silage & Lactobacillus plantarum CGMCC 8198 & $35-39$ & ND & ND & Gu et al. (2014) \\
\hline Silage & Lactobacillus plantarum Lp09 AND Lp45 & ND & ND & ND & Huang et al. (2013) \\
\hline Kefir grains & Lactobacillus plantarum BBE7 & 43 & ND & ND & Dong et al. (2012) \\
\hline Soil & Xanthomonas maltophilia CBS 827.97 & 52 & $7.9-8.5$ & $25-40$ & Dean et al. (2002) \\
\hline
\end{tabular}

${ }^{a}$ Molecular mass of tetramer.

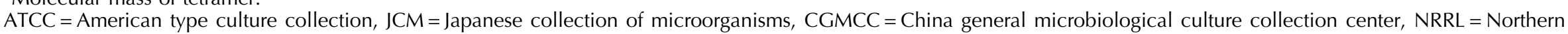
regional research laboratory, the agricultural research service culture collection, ND = not determined. 
2014b). Recent probiotics studies have already shown that oral administration of BSH-producing lactobacilli could affect lipid metabolism, consequently reducing body weight and/or cholesterol level in human beings (Jones et al., 2013), rats (Pato et al., 2004; Kumar et al., 2011), mice (Park et al., 2013, 2014; Miyoshi et al., 2014), and pigs (De Smet et al., 1998).

Molecular and cellular studies also provided new insights into underlying mechanisms of the effect of BSH enzyme on host lipid metabolism and energy harvest. Clearly, unconjugated bile acids, directly resulting from $\mathrm{BSH}$ activity, are less effective than conjugated bile acids in the emulsification of dietary fat and consequently affect lipid absorption and metabolism. However, unconjugated bile acids could exert more profound impacts on host energy harvest both locally and systemically. Farnesoid X receptor (FXR), which is preferentially stimulated by unconjugated bile acids, not only regulate lipogenesis and triglyceride synthesis (Watanabe et al., 2004; Li et al., 2013), but also regulate glucose homeostasis by increasing glycogen synthesis (Zhang et al., 2006; Caron et al., 2013) or decreasing glycolysis (Caron et al., 2013). Using a pig model, Pereira-Fantini et al. (2014) examined the impact of BSH-mediated bile acid dysmetabolism on FXR signaling pathways and clinical outcomes and showed that alterations in bile acid composition may have contributed to the observed disturbance in FXR-mediated signaling pathways (Pereira-Fantini et al., 2014).

Notably, obesity development is a complex physiological issue. The BSH-mediated bile salt metabolism is only one of several potential mechanisms by which the microbiota affect host energy harvest and weight gain (Walker and Parkhill, 2013). The studies described above only provide indirect evidence supporting the role of $\mathrm{BSH}$-producing probiotics or BSH-mediated bile metabolism in host lipid metabolism and energy harvest. Direct and controlled approaches are required in order to obtain complete understanding of BSH-mediated regulation of host weight gain and lipid metabolism.

Recently, using a controlled system in conjunction with a mouse model, Joyce et al. (2014a) obtained the first direct evidence demonstrating that manipulation of in situ $\mathrm{BSH}$ activity alone significantly influenced lipid metabolism, signaling functions, and weight gain (Joyce et al., 2014a). Briefly, two well characterized L. salivarius BSH enzymes were cloned into an E. coli host strain (MG1655). The recombinant Escherichia coli constructs could effectively colonize the gastrointestinal tract of mice with expression of high level of $\mathrm{BSH}$ activity. Colonization of germ-free mice with such BSH-producing E. coli strain elevated intestinal BSH activity and resulted in local bile acids deconjugation with concomitant reduced levels in body weight and cholesterol, alternations in lipid metabolism, signaling functions, local and systemic transcriptome profiles in the pathways governing lipid metabolism (Joyce et al., 2014a). Notably, in conventionally raised mice, enhanced in situ BSH activity also caused local bile acid deconjugation, reduced mouse weight gain, lowered serum cholesterol level, and reduced liver triglyceride level, which further demonstrates that $\mathrm{BSH}$ is a key mechanism through which the microbiota modulates host lipid metabolism and dietary energy harvest (Joyce et al., 2014a). In addition to its ability to alter local (gastrointestinal) functions, BSH activity could systemically affect host physiology such that the BSH activity-mediated bile acids can interact with transporters (e.g. $A b c g 5 / 8$ ) and regulators (e.g. FXR regulon, Fiaf) that lead to change in body mass (Joyce et al., 2014a).

\section{Other physiological process}

The BSH-mediated unconjugated bile acids also affect immune homeostasis because of their ability to modulate a panel of effectors in the intestine, such as inducible nitric oxide synthase (iNOS) (Inagaki et al., 2006), the antimicrobial peptide RegIII $\gamma$ produced by intestinal paneth cells (Joyce et al., 2014a), and dendritic cell differentiation (Ichikawa et al., 2012; Joyce et al., $2014 b)$. In addition to the pathway via intestinal FXR, unconjugated bile acids also affect TGR5-mediated adipose tissue development and weight loss (Watanabe et al., 2006; Svensson et al., 2013). Interestingly, Joyce et al. (2014a) also observed that enhanced in situ BSH activity reversed the expression pattern of genes responsible for regulating circadian rhythm (e.g., $D b p$ ) and other genes central to circadian clock (Joyce et al., 2014a). Finally, unconjugated bile acids can also alter intestinal microbiota, consequently may exert more complex impacts on host (Inagaki et al., 2006; Islam et al., 2011).

\section{Potential adverse effects due to high-level BSH activity in the intestine}

High-level BSH activity would result in a large proportion of unconjugated bile acids, which can lead to malabsorption of lipid and may cause steatorrhea in the host (Kim and Lee, 2005). Recent research also indicated that deconjugation of bile salts by BSH-producing lactobacilli is an important factor leading to short bowel syndrome due to abnormal lipid metabolism and a disrupted bile acid profile (Bongaerts et al., 2000; Choi et al., 2014).

BSH-mediated deconjugation of bile salts can increase bile recovery from passive absorption across the colonic epithelium by making bile salts more hydrophobic, which may also cause some adverse effects. For example, a high concentration of secondary bile acids in blood and feces, that are produced by a multistep of $7 \alpha$-dehydroxylation reaction from unconjugated bile acids, are proposed to be related to the pathogenesis of cholesterol gallstone diseases as well as colon cancer (van Faassen et al., 1987; Färkkilä and Miettinen, 1990; Marteau and Rambaud, 1993; McGarr et al., 2005; Venneman and van Erpecum, 2010; Ou et al., 2013). Secondary bile acids may increase the risk of cancer by increasing oxidative stress and associated DNA damage (Cooke et al., 2003; Bernstein et al., 2005). The sulfonic acid moiety in unconjugated bile acids could be reduced and dissimilated to hydrogen sulfide, which is highly toxic and can increase colon cell turnover (Christl et al., 1996; Corzo and Gilliland, 1999; Lie et al., 1999; Laue et al., 2001; Ridlon et al., 2006). Hydrogen sulfide is a potent inhibitor of colonic butyrate metabolism, which is a key nutrient and regulator of cell turnover (Christl et al., 1996; Van Eldere et al., 1996). 
Hydrogen sulfide can also reduce apoptosis in colon cancer cells by preventing the function of a chemo-preventative agent $\beta$-phenylethyl isothiocyanate (PEITC) (Rose et al., 2005).

\section{Target BSH for enhanced animal production and health}

In contrast to the significant progress on $\mathrm{BSH}$ research for human health described above, little information exists concerning BSH and BSH-producing bacteria in food animals. Some early studies evaluated direct usage of bile salts as a feed additive to improve feed efficiency due to the well-recognized role of bile salts in fat digestion (Kussaibati et al., 1982; Reinhart et al., 1988). In chickens, supplementation of bile salts in the diet increased the absorption of fatty acids, but had no influence on chickens with fat-free diet (Kussaibati et al., 1982). Presence of bile salts in the diet also increased fat digestibility in swine after the weaning period (Reinhart et al., 1988). Although the findings from these studies are encouraging, bile salts have not been adopted by the feed industry as feed additives to improve growth performance of food animals, likely due to the issues of cost, availability, and complex biotransformation of bile salts in the gastrointestinal tract.

AGPs are defined as a group of antibiotics used in feed at sub-therapeutic level to improve average daily weight gain and feed efficiency in food animals. This husbandry technique has been practiced since the 1950s. However, use of AGPs has been associated with the emergence of antibiotic-resistant human pathogens of animal origins. Therefore, ending the use of AGPs is a worldwide trend to protect public health. Effective alternatives to AGPs are urgently needed to maintain current animal production levels without threatening public health. Recent animal studies on the effect of AGP usage on intestinal microbiome indicate that the enhanced feed efficiency and body weight gain in food animals due to AGP usage is inversely related to the BSH activity as well as the abundance of potent BSH-producers in the intestine (Lin, 2014).

As early as in 1980s, Feighner and Dashkevicz (1987) reported that use of AGP reduced intestinal BSH activity in poultry and they proposed that inhibition of $\mathrm{BSH}$ activity would promote feed efficiency and weight gain in food animals. In this early study, a radiochemical method was successfully developed to directly determine BSH activity in intestinal contents; however, the method used in this study was technically challenging and time consuming (Feighner and Dashkevicz, 1987). Notably, the standard BSH activity assay widely used is not feasible for examining fecal BSH activity because of the high levels of background caused by free amino acids in intestinal contents. To date, fecal bile acid profile is an acceptable indicator for evaluating BSH activity in the intestinal contents. Consistent with the finding by Feighner and Dashkevicz (1987), Knarreborg et al. (2004) also observed AGP usage reduced concentration of unconjugated bile salts in the intestine of broilers by using reversed-phase HLPC method, which led to an enhanced bioavailability of $\alpha$-tocopheryl acetate. In multiple pen trials, Guban et al. (2006) further confirmed that AGP treatment improved weight gain and fat digestibility in broilers, decreased population levels of $L$. salivarius, and significantly reduced $\mathrm{BSH}$ activity in the intestine, which was reflected by a decreased pool of deconjugated bile salts in ileal contents using a HPLC method. In pigs, De Smet et al. (1998) observed that oral administration of the $L$. reuteri with $\mathrm{BSH}$ activity influenced host lipid metabolism and decreased total and LDL-cholesterol concentrations. Du Toit et al. (1998) also had a similar finding in a minipig feeding trial using BSH-positive probiotic mix. However, both of these pig studies (De Smet et al., 1998; Du Toit et al., 1998) lack determination of intestinal BSH activity, which is needed to rule out potential pleotropic effects resulting from the treatment with $\mathrm{BSH}$-producing probiotics.

Regarding response of intestinal microbiota to AGPs, a key issue for us to understand the mode of action of AGP, cultureindependent molecular approaches have been used to examine the effect of AGPs on intestinal microbiota in poultry and swine; to date, more than ten papers have been published in this field (Lin, 2014). Not surprisingly, long-term supplementation of diet with AGPs significantly affected the microbial ecology in the intestine in all reported studies. However, the specific bacteria or environmental niche changes that are meaningful and are linked to the desired phenotype of enhanced growth performance need to be clarified. In-depth comparative analysis of these animal microbiome studies led to an interesting finding: in most chicken and swine studies, use of AGP reduced the population of Lactobacillus species, the major BSH-producing bacteria in the animal intestine (Begley et al., 2006; Lin, 2014). The independent findings from these food animal studies, together with those from human BSH research summarized above, are like jigsaw pieces which seem to be scattered but are in fact tightly interrelated. Therefore, it was proposed that $\mathrm{BSH}$ is a key mechanistic microbiome target for developing novel alternatives to AGPs and this hypothesis prompted us to identify and characterize a potent BSH enzyme from a chicken L. salivarius probiotic strain (Wang et al., 2012). Interestingly, copper and zinc compounds displayed a potent inhibitory effect on BSH enzyme activity in this study, which not only provides scientific evidence to understand the mode of action of high dietary concentrations of copper/zinc for growth promotion, but also strongly supports our hypothesis that BSH inhibitors may serve as promising alternatives to AGPs (Wang et al., 2012). Subsequently, by taking advantage of the unique feature of the L. salivarius BSH enzyme (Wang et al., 2012), an efficient high-throughput screening system was successfully developed and used to discover BSH inhibitors (Smith et al., 2014). Unlike many BSH enzymes from other bacteria that have narrow substrate spectrum, the $L$. salivarius $\mathrm{BSH}$ displayed a potent hydrolysis activity towards both glycol-conjugated and taurineconjugated bile salts. The broad substrates specificity nature of this BSH makes it an ideal candidate for screening desired BSH inhibitors. This hypothesis is further tested by our recent study showing the identified BSH inhibitors also exhibited potent inhibitory effects on a phylogenetically distant BSH from L. acidophilus (Lin et al., 2014).

Despite the recognized AMR issues associated with antibiotic usage in food animals, animal industries still heavily rely on 
antibiotics due to the lack of practical and consistent antibiotic alternative approaches. Solely limiting antibiotics without providing effective alternatives would compromise animal production and health. BSH inhibitors are promising alternatives to AGPs for enhanced feed efficiency and growth performance. Successful development of effective non-antibiotic BSH inhibitor feed additives could reduce the dependence on in-feed antibiotics for growth promotion, consequently mitigating AMR pressure in agriculture ecosystems, a significant and timely issue impacting animal health and food safety.

Other types of antibiotic-alternative products, such as probiotics, prebiotics, and organic acids, have drawn wide attention and have been developed and used to alter intestinal microbiota for improving animal health and production (Dibner and Richards, 2005; Lin, 2014). However, very limited data are available to scientifically justify the choice of specific bacterial species or products for growth promotion and results are inconsistent from independent studies (Dibner and Richards, 2005). For example, although probiotics containing Lactobacillus are well recognized for their beneficial effects on boosting host immunity, these probiotics could have a negative impact on host lipid metabolism due to $\mathrm{BSH}$ production. Specifically, in a large pen trial, Sharifi et al. (2012) observed that supplementation of a 7-bacterial species probiotic (Protexin) to fat-rich diets significantly reduced body weight gain, fat digestibility, and feed conversion in broilers. Moreover, using a different 5-bacterial species competitive exclusion probiotic product, Mountzouris et al. (2010) also observed similar inferior feed conversion efficiency and reduced fat digestibility in response to probiotic treatment in broilers. These investigators have proposed that the enrichment of the intestinal microflora, particularly lactobacilli, due to probiotic supplementation caused enhanced BSH activity in the intestine, leading to detrimental effects on lipid metabolism and growth performance of broilers. Therefore, improved knowledge in the role of BSH and BSH-producing bacteria will help design rationally tailored probiotics that will enhance animal health and performance. For example, the BSH inhibitors could also be used together with certain $\mathrm{BSH}$-producing probiotics to maximize the beneficial effect of the probiotics by mitigating their potential negative impact on host fat digestion. This approach may further help animal production industries optimize existing probiotic and prebiotic additives for enhanced feed efficiency, growth performance and profitability.

\section{Conclusions and research gaps}

Antibiotics have been heavily used for animal farming to maintain animal production and health. However, farm use of antibiotics is a driving force to enrich AMR genes (called the 'resistome') in various niches and to promote pools of resistant pathogenic bacteria, raising food safety and public health concerns (Davies, 2014; Perry et al., 2014). To effectively mitigate AMR in agricultural systems, a reduction in the use of antibiotics in farming is imperative. Thus, intensive efforts are critically needed to develop effective non-antibiotic growth promotion strategies that can be practically implemented by animal producers. Recent microbiome studies have provided compelling evidence that $\mathrm{BSH}$ is a key mechanistic microbiome target for developing novel alternatives to AGPs. Development of $\mathrm{BSH}$ inhibitor-based non-antibiotic feed additives directly addresses the nutrition concern (feed efficiency/growth rate) that prevents animal industries from reducing antibiotic usage. In addition to benefitting healthy animals under routine management, the weight-enhancing BSH inhibitors may also help sick animals better harvest dietary energy while combatting infectious diseases or environmental/production stress.

Despite the significant role of bacterial BSH activity in host lipid metabolism and energy harvest, research on BSH is still in its infancy. In particular, little effort has been placed on characterization of $\mathrm{BSH}$ enzymes and/or BSH-producing bacteria in food animals. Several significant gaps remain in knowledge associated with BSH in food animal production and health. Filling these gaps will not only directly benefit animal health but also provide insights and likely new model systems for human health research, leading to novel 'One Health' measures for enhanced animal production, food safety, and human nutrition.

- Ecology of BSH enzymes and BSH-producing bacteria in the intestine. To date, only a limited number of BSH enzymes have been identified in the intestinal bacteria isolated from food animals (Table 1). With the aid of next generation sequencing technologies and bioinformatics tools, functional and comparative metagenomic analyses of intestinal $\mathrm{BSH}$ in food animals are warranted and will provide a better picture of the diversity and function of $\mathrm{BSH}$ in the intestine. Information in conjunction with other phenotypic examinations would improve our understanding on the role of $\mathrm{BSH}$ in the symbiotic relationship between the gastrointestinal microbiome and animal host. Given that specific BSH enzyme(s) and corresponding BSH-producing bacteria may serve as biomarkers for health statuses of animal hosts, understanding the ecology of BSH enzymes and BSH-producing bacteria in the intestine would facilitate the development of diagnostics to evaluate the health status of animals and people.

- Comprehensive evaluation using a controlled system together with a new model system is still critically needed to provide new mechanistic information for the role of BSH in host energy harvest and weight gain. Given the increasing awareness of important roles of microbiota in intestine health, development of specifically tailored probiotics is a logical strategy for practical application, but this approach needs an in-depth understanding of the molecular, physiological, and ecological features of probiotic organisms in order to select and design probiotics for safe, effective administration for specific purposes. To date, there are not any studies using BSH-negative and $\mathrm{BSH}$-overproducing probiotic organisms to definitively link BSH activity to the specific phenotype and their impacts on host animals and native microbiomes. This is likely due to the challenge for manipulating $\mathrm{BSH}$ activity in commensal organisms for specific laboratory animal hosts and to the lack of public acceptance of using genetically modified organisms (GMOs) in human trials. While this concern has been partly addressed with a recent E. coli knock-in model (Joyce et al., 
2014a), manipulating BSH activity of a natural intestinal commensal organism in an animal model would be a better approach. Recent characterization of $L$. salivarius as a potent BSH producer (Wang et al., 2012) provides an excellent opportunity to address this issue using a food animal model system, because genetic tools to manipulate $L$. salivarius have been well established. Such research efforts would enable us to better manage body weight by manipulating microbiota in people and animals.

- Developing alternatives to AGPs by inhibiting BSH activity in the intestine. In addition to discovering more novel BSH inhibitors, comprehensive animal trials are essential to further evaluate and select desired BSH inhibitors. It is likely that prolonged use of a particular BSH inhibitor could lead to negative physiological consequences due to pleotropic effects of specific inhibitor and complexity of host physiology. For example, because $\mathrm{BSH}$ inhibitors are expected to improve lipid metabolism, it is important to examine if energy harvest and weight gain is partitioned adequately and not skewed toward excess fat deposition, which would be undesirable for both animal producers and consumers. In addition, it is also warranted to examine how inhibition of BSH activity affects the bile profile, as well as the gastrointestinal microbial community and all the implications that these changes hold for animal health and productivity.

- Structural basis of BSH function. Given ecological diversity of $\mathrm{BSH}$ in the intestinal microbiome, structure analyses of $\mathrm{BSH}$ enzymes from various species are highly warranted, which would reveal critical residues in catalysis and provide key information on the substrates selectivity of BSH enzymes. Clearly, such basic studies also will directly facilitate future translational research, such as using molecular docking to develop desired $\mathrm{BSH}$ inhibitors for growth promotion in food animals.

\section{Acknowledgment}

Work in our laboratory was supported by a University of Tennessee AgResearch Innovation Grant and a University of Tennessee Research Foundation Technology Maturation Fund.

\section{References}

Appleby RN and Walters JR (2014). The role of bile acids in functional GI disorders. Neurogastroenterology \& Motility 26: 1057-1069.

Aries V and Hill M (1970). Degradation of steroids by intestinal bacteria I. Deconjugation of bile salts. Biochimica et Biophysica Acta (BBA)-Lipids and Lipid Metabolism 202: 526-534.

Batta A, Salen G, Arora R, Shefer S, Batta M and Person A (1990). Side chain conjugation prevents bacterial 7-dehydroxylation of bile acids. Journal of Biological Chemistry 265: 10925-10928.

Begley M, Gahan CG and Hill C (2005). The interaction between bacteria and bile. FEMS Microbiology Reviens 29: 625-651.

Begley M, Hill C and Gahan CG (2006). Bile salt hydrolase activity in probiotics. Applied and Environmental Microbiology 72: 1729-1738.

Bernstein H, Bernstein C, Payne C, Dvorakova K and Garewal H (2005). Bile acids as carcinogens in human gastrointestinal cancers. Mutation Research/Reviews in Mutation Research 589: 47-65.

Bi J, Fang F, Lu S, Du G and Chen J (2013). New insight into the catalytic properties of bile salt hydrolase. Journal of Molecular Catalysis B: Ensymatic 96: 46-51.
Bongaerts GP, Severijnen RS, Tangerman A, Verrips A and Tolboom JJ (2000). Bile acid deconjugation by Lactobacilli and its effects in patients with a short small bowel. Journal of Gastroenterology 35: 801-804.

Camilleri M and Gores GJ (2015). Therapeutic targeting of bile acids. American Journal of Physiology-Gastrointestinal and Liver Physiology 309: G209-G215.

Caron S, Samanez CH, Dehondt H, Ploton M, Briand O, Lien F, Dorchies E, Dumont J, Postic C and Cariou B (2013). Farnesoid $\mathrm{X}$ receptor inhibits the transcriptional activity of carbohydrate response element binding protein in human hepatocytes. Molecular and Cellular Biology 33: 2202-2211.

Chae J, Valeriano V, Kim GB and Kang DK (2013). Molecular cloning, characterization and comparison of bile salt hydrolases from Lactobacillus johnsonii PF01. Journal of Applied Microbiology 114: 121-133.

Choi S-B, Lew L-C, Yeo S-K, Nair Parvathy S and Liong M-T (2014). Probiotics and the BSH-related cholesterol lowering mechanism: a Jekyll and Hyde scenario. Critical Reviens in Biotechnology 35: 392-401.

Christl SU, Eisner H-D, Dusel G, Kasper H and Scheppach W (1996). Antagonistic effects of sulfide and butyrate on proliferation of colonic mucosa. Digestive Diseases and Sciences 41: 2477-2481.

Claesson MJ, Li Y, Leahy S, Canchaya C, van Pijkeren JP, Cerdeño-Tárraga AM, Parkhill J, Flynn S, O’Sullivan GC and Collins JK (2006). Multireplicon genome architecture of Lactobacillus salivarius. Proceedings of the National Academy of Sciences of the United States of America 103: 6718-6723.

Coleman JP and Hudson LL (1995). Cloning and characterization of a conjugated bile acid hydrolase gene from Clostridium perfringens. Applied and Environmental Microbiology 61: 2514-2520.

Cooke MS, Evans MD, Dizdaroglu M and Lunec J (2003). Oxidative DNA damage: mechanisms, mutation, and disease. FASEB Journal 17: 1195-1214.

Corzo G and Gilliland S (1999). Bile salt hydrolase activity of three strains of Lactobacillus acidophilus. Journal of Dairy Science 82: 472-480.

Damodharan K, Lee YS, Palaniyandi SA, Yang SH and Suh J-W (2015). Preliminary probiotic and technological characterization of Pediococcus pentosaceus strain KID7 and in vivo assessment of its cholesterol-lowering activity. Frontiers in Microbiology 6: 768. doi: 10.3389/fmicb.2015.00768.

Davies J (2014). Antibiotic resistance in and from nature. In: Atlas RM and Maloy S (eds) One Healtb: People, Animals, and the Environment. Washington, DC: American Society for Microbiology, pp. 185-194.

De Aguiar Vallim TQ, Tarling EJ and Edwards PA (2013). Pleiotropic roles of bile acids in metabolism. Cell Metabolism 17: 657-669.

Dean M, Cervellati C, Casanova E, Squerzanti M, Lanzara V, Medici A, de Laureto PP and Bergamini CM (2002). Characterization of cholylglycine hydrolase from a bile-adapted strain of Xanthomonas maltophilia and its application for quantitative hydrolysis of conjugated bile salts. Applied and Environmental Microbiology 68: 3126-3128.

De Smet I, De Boever P and Verstraete W (1998). Cholesterol lowering in pigs through enhanced bacterial bile salt hydrolase activity. British Journal of Nutrition 79: 185-194.

Dibner J and Richards J (2005). Antibiotic growth promoters in agriculture: history and mode of action. Poultry Science 84: 634-643.

Dong Z, Zhang J, Lee B, Li H, Du G and Chen J (2012). A bile salt hydrolase gene of Lactobacillus plantarum BBE7 with high cholesterolremoving activity. European Food Research and Technology 235: 419-427.

Dussurget O, Cabanes D, Dehoux P, Lecuit M, Buchrieser C, Glaser P and Cossart P (2002). Listeria monocytogenes bile salt hydrolase is a PrfA-regulated virulence factor involved in the intestinal and hepatic phases of listeriosis. Molecular Microbiology 45: 1095-1106.

Du Toit M, Franz C, Dicks L, Schillinger U, Haberer P, Warlies B, Ahrens F and Holzapfel W (1998). Characterisation and selection of probiotic lactobacilli for a preliminary minipig feeding trial and their effect on serum cholesterol levels, faeces $\mathrm{pH}$ and faeces moisture content. International Journal of Food Microbiology 40: 93-104.

Evans MJ, Mahaney PE, Borges-Marcucci L, Lai K, Wang S, Krueger JA, Gardell SJ, Huard C, Martinez R and Vlasuk GP (2009). A 
synthetic farnesoid X receptor (FXR) agonist promotes cholesterol lowering in models of dyslipidemia. American Journal of Physiology-Gastrointestinal and Liver Physiology 296: G543-G552.

Fang F, Li Y, Bumann M, Raftis EJ, Casey PG, Cooney JC, Walsh MA and O'Toole PW (2009). Allelic variation of bile salt hydrolase genes in Lactobacillus salivarius does not determine bile resistance levels. Journal of Bacteriology 191: 5743-5757.

Färkkilä M and Miettinen TA (1990). Lipid metabolism in bile acid malabsorption. Annals of Medicine 22: 5-13.

Feighner SD and Dashkevicz MP (1987). Subtherapeutic levels of antibiotics in poultry feeds and their effects on weight gain, feed efficiency, and bacterial cholyltaurine hydrolase activity. Applied and Environmental Microbiology 53: 331-336.

Feighner SD and Dashkevicz MP (1988). Effect of dietary carbohydrates on bacterial cholyltaurine hydrolase in poultry intestinal homogenates. Applied and Environmental Microbiology 54: 337-342.

Gopal-Srivastava R and Hylemon PB (1988). Purification and characterization of bile salt hydrolase from Clostridium perfringens. Journal of Lipid Research 29: 1079-1085.

Grill J, Schneider F, Crociani J and Ballongue J (1995). Purification and characterization of conjugated bile salt hydrolase from Bifidobacterium longum BB536. Applied and Environmental Microbiology 61: 2577-2582.

Gu X-C, Luo X-G, Wang C-X, Ma D-Y, Wang Y, He Y-Y, Li W, Zhou $\mathrm{H}$ and Zhang T-C (2014). Cloning and analysis of bile salt hydrolase genes from Lactobacillus plantarum CGMCC No. 8198. Biotechnology Letters 36: 975-983.

Guban J, Korver D, Allison G and Tannock G (2006). Relationship of dietary antimicrobial drug administration with broiler performance, decreased population levels of Lactobacillus salivarius, and reduced bile salt deconjugation in the ileum of broiler chickens. Poultry Science 85: 2186-2194.

Gupta S, Stravitz RT, Dent P and Hylemon PB (2001). Down-regulation of cholesterol $7 \alpha$-hydroxylase (CYP7A1) gene expression by bile acids in primary rat hepatocytes is mediated by the c-Jun $\mathrm{N}$-terminal kinase pathway. Journal of Biological Chemistry 276: 15816-15822.

Hofmann AF and Mysels KJ (1992). Bile acid solubility and precipitation in vitro and in vivo: the role of conjugation, $\mathrm{pH}$, and $\mathrm{Ca} 2+$ ions. Journal of Lipid Research 33: 617-626.

Houten SM, Watanabe M and Auwerx J (2006). Endocrine functions of bile acids. EMBO Journal 25: 1419-1425.

Huang Y, Wang X, Wang J, Wu F, Sui Y, Yang L and Wang Z (2013). Lactobacillus plantarum strains as potential probiotic cultures with cholesterol-lowering activity. Journal of Dairy Science 96: 2746-2753.

Ichikawa R, Takayama T, Yoneno K, Kamada N, Kitazume MT, Higuchi $\mathrm{H}$, Matsuoka $\mathrm{K}$, Watanabe $\mathrm{M}$, Itoh $\mathrm{H}$ and Kanai $\mathrm{T}$ (2012). Bile acids induce monocyte differentiation toward interleukin-12 hypo-producing dendritic cells via a TGR5-dependent pathway. Immunology 136: 153-162.

Inagaki T, Moschetta A, Lee Y-K, Peng L, Zhao G, Downes M, Ruth TY, Shelton JM, Richardson JA and Repa JJ (2006). Regulation of antibacterial defense in the small intestine by the nuclear bile acid receptor. Proceedings of the National Academy of Sciences of the United States of America 103: 3920-3925.

Islam KS, Fukiya S, Hagio M, Fujii N, Ishizuka S, Ooka T, Ogura Y, Hayashi T and Yokota A (2011). Bile acid is a host factor that regulates the composition of the cecal microbiota in rats. Gastroenterology 141: 1773-1781.

Jiang J, Hang X, Zhang M, Liu X, Li D and Yang H (2010). Diversity of bile salt hydrolase activities in different lactobacilli toward human bile salts. Annals of Microbiology 60: 81-88.

Johnson L (1998). Bile secretion and gallbladder function. Essential Medical Physiology 2: 465-471.

Jones BV, Begley M, Hill C, Gahan CG and Marchesi JR (2008). Functional and comparative metagenomic analysis of bile salt hydrolase activity in the human gut microbiome. Proceedings of the National Academy of Sciences of the United States of America 105: 13580-13585.
Jones ML, Tomaro-Duchesneau C, Martoni CJ and Prakash S (2013). Cholesterol lowering with bile salt hydrolase-active probiotic bacteria, mechanism of action, clinical evidence, and future direction for heart health applications. Expert Opinion on Biological Therapy 13: 631-642.

Jones ML, Martoni CJ, Ganopolsky JG, Labbé A and Prakash S (2014). The human microbiome and bile acid metabolism: dysbiosis, dysmetabolism, disease and intervention. Expert Opinion on Biological Therapy 14: 467-482.

Joyce SA, MacSharry J, Casey PG, Kinsella M, Murphy EF, Shanahan F, Hill C and Gahan CG (2014a). Regulation of host weight gain and lipid metabolism by bacterial bile acid modification in the gut. Proceedings of the National Academy of Sciences of the United States of America 111: 7421-7426.

Joyce SA, Shanahan F, Hill C and Gahan CG (2014b). Bacterial bile salt hydrolase in host metabolism: potential for influencing gastrointestinal microbe-host crosstalk. Gut Microbes 5: 669-674.

Kahn SE, Hull RL and Utzschneider KM (2006). Mechanisms linking obesity to insulin resistance and type 2 diabetes. Nature 444: 840-846.

Kawamoto K, Horibe I and Uchida K (1989). Purification and characterization of a new hydrolase for conjugated bile acids, chenodeoxycholyltaurine hydrolase, from Bacteroides vulgatus. Journal of Biochemistry 106: 1049-1053.

Kim G and Lee BH (2005) Biochemical and molecular insights into bile salt hydrolase in the gastrointestinal microflora-a review. Asian Australasian Journal of Animal Sciences 18: 1505.

Kim G-B, Miyamoto CM, Meighen EA and Lee BH (2004). Cloning and characterization of the bile salt hydrolase genes (bsh) from Bifidobacterium bifidum strains. Applied and Environmental Microbiology 70: 5603-5612.

Kim G-B, Brochet M and Lee BH (2005). Cloning and characterization of a bile salt hydrolase (bsh) from Bifidobacterium adolescentis. Biotechnology Letters 27: 817-822.

Knarreborg A, Lauridsen C, Engberg RM and Jensen SK (2004). Dietary antibiotic growth promoters enhance the bioavailability of $\alpha$-tocopheryl acetate in broilers by altering lipid absorption. Journal of Nutrition 134: 1487-1492.

Kumar R, Grover S and Batish VK (2011). Hypocholesterolaemic effect of dietary inclusion of two putative probiotic bile salt hydrolaseproducing Lactobacillus plantarum strains in Sprague-Dawley rats. British Journal of Nutrition 105: 561-573.

Kumar RS, Brannigan JA, Prabhune AA, Pundle AV, Dodson GG, Dodson EJ and Suresh C (2006). Structural and functional analysis of a conjugated bile salt hydrolase from Bifidobacterium longum reveals an evolutionary relationship with penicillin $\mathrm{V}$ acylase. Journal of Biological Chemistry 281: 32516-32525.

Kussaibati R, Guillaume J and Leclercq B (1982). The effects of endogenous energy, type of diet, and addition of bile salts on true metabolizable energy values in young chicks. Poultry Science 61: 2218-2223.

Lambert JM, Bongers RS, de Vos WM and Kleerebezem M (2008). Functional analysis of four bile salt hydrolase and penicillin acylase family members in Lactobacillus plantarum WCFS1. Applied and Environmental Microbiology 74: 4719-4726.

Laue H, Friedrich M, Ruff J and Cook AM (2001). Dissimilatory sulfite reductase (desulfoviridin) of the taurine-degrading, non-sulfatereducing bacterium Bilophila wadsworthia RZATAU contains a fused DsrB-DsrD subunit. Journal of Bacteriology 183: 1727-1733.

Lepercq P, Relano P, Cayuela C and Juste C (2004). Bifidobacterium animalis strain DN-173 010 hydrolyses bile salts in the gastrointestinal tract of pigs. Scandinavian Journal of Gastroenterology 39: 1266-1271.

Li F, Jiang C, Krausz KW, Li Y, Albert I, Hao H, Fabre KM, Mitchell JB, Patterson AD and Gonzalez FJ (2013). Microbiome remodelling leads to inhibition of intestinal farnesoid $\mathrm{X}$ receptor signalling and decreased obesity. Nature Communications 4: 2384. doi: $10.1038 /$ ncomms 3384 .

Li Y, Raftis E, Canchaya C, Fitzgerald GF, van Sinderen D and O'Toole PW (2006). Polyphasic analysis indicates that Lactobacillus salivarius 
subsp. salivarius and Lactobacillus salivarius subsp. salicinius do not merit separate subspecies status. International Journal of Systematic and Evolutionary Microbiology 56: 2397-2403.

Lie TJ, Clawson ML, Godchaux W and Leadbetter ER (1999). Sulfidogenesis from 2-aminoethanesulfonate (taurine) fermentation by a morphologically unusual sulfate-reducing bacterium, Desulforhopalus singaporensis sp. nov. Applied and Environmental Microbiology 65: 3328-3334.

Lin J (2011). Effect of antibiotic growth promoters on intestinal microbiota in food animals: a novel model for studying the relationship between gut microbiota and human obesity? Frontiers in Cellular and Infection Microbiology 2: 53. doi: 10.3389/ fmicb.2011.00053.

Lin J (2014). Antibiotic growth promoters enhance animal production by targeting intestinal bile salt hydrolase and its producers. Frontiers in Microbiology 5: 33. doi: 10.3389/fmicb.2014.00033.

Lin J, Negga R, Zeng X and Smith K (2014). Effect of bile salt hydrolase inhibitors on a bile salt hydrolase from Lactobacillus acidophilus. Pathogens 3: 947-956.

Liong $\mathrm{M}$ and Shah N (2005). Acid and bile tolerance and cholesterol removal ability of lactobacilli strains. Journal of Dairy Science 88: $55-66$.

Lundeen SG and Savage DC (1990). Characterization and purification of bile salt hydrolase from Lactobacillus sp. strain 100-100. Journal of Bacteriology 172: 4171-4177.

Marteau P and Rambaud J-C (1993). Potential of using lactic acid bacteria for therapy and immunomodulation in man. FEMS Microbiology Reviews 12: 207-220.

Masuda N (1981). Deconjugation of bile salts by Bacteroides and Clostridium. Microbiology and Immunology 25: 1-11.

McAuliffe O, Cano RJ and Klaenhammer TR (2005). Genetic analysis of two bile salt hydrolase activities in Lactobacillus acidophilus NCFM. Applied and Environmental Microbiology 71: 4925-4929.

McGarr SE, Ridlon JM and Hylemon PB (2005). Diet, anaerobic bacterial metabolism, and colon cancer: a review of the literature. Journal of Clinical Gastroenterology 39: 98-109.

Miyoshi M, Ogawa A, Higurashi S and Kadooka Y (2014). Anti-obesity effect of Lactobacillus gasseri SBT2055 accompanied by inhibition of pro-inflammatory gene expression in the visceral adipose tissue in diet-induced obese mice. European Journal of Nutrition 53: 599-606.

Mountzouris K, Tsitrsikos P, Palamidi I, Arvaniti A, Mohnl M, Schatzmayr G and Fegeros K (2010). Effects of probiotic inclusion levels in broiler nutrition on growth performance, nutrient digestibility, plasma immunoglobulins, and cecal microflora composition. Poultry Science 89: 58-67.

Mukherji R and Prabhune A (2015). Possible correlation between bile salt hydrolysis and AHL deamidation: Staphylococcus epidermidis RM1, a potent quorum quencher and bile salt hydrolase producer. Applied Biochemistry and Biotechnology 176: 140-150.

Nair P, Gordon M and Reback J (1967). The enzymatic cleavage of the carbon-nitrogen bond in $3 \alpha, 7 \alpha, 12 \alpha$-trihydroxy-5 $\beta$-cholan-24oylolycine. Journal of Biological Chemistry 242: 7-11.

Oh H-K, Lee JY, Lim SJ, Kim MJ, Kim G-B, Kim JH, Hong S-K and Kang D-K (2008). Molecular cloning and characterization of a bile salt hydrolase from Lactobacillus acidophilus PF01. Journal of Microbiology and Biotecbnology 18: 449-456.

Ou J, Carbonero F, Zoetendal EG, DeLany JP, Wang M, Newton K, Gaskins HR and O'Keefe SJ (2013). Diet, microbiota, and microbial metabolites in colon cancer risk in rural Africans and African Americans. American Journal of Clinical Nutrition 98: 111-120.

Park D-Y, Ahn Y-T, Park S-H, Huh C-S, Yoo S-R, Yu R, Sung M-K, McGregor RA and Choi M-S (2013). Supplementation of Lactobacillus curvatus HY7601 and Lactobacillus plantarum KY1032 in diet-induced obese mice is associated with gut microbial changes and reduction in obesity. PloS ONE 8: e59470.

Park JE, Oh SH and Cha YS (2014). Lactobacillus plantarum LG42 isolated from gajami sik-hae decreases body and fat pad weights in diet-induced obese mice. Journal of Applied Microbiology 116: $145-156$.
Patel AK, Singhania RR, Pandey A and Chincholkar SB (2010). Probiotic bile salt hydrolase: current developments and perspectives. Applied Biochemistry and Biotechnology 162: 166-180.

Pato U, Surono IS and Hosono A (2004). Hypocholesterolemic effect of indigenous dadih lactic acid bacteria by deconjugation of bile salts. Asian-Australasian Journal of Animal Sciences 17: 1741-1745.

Pavlović N, Stankov K and Mikov M (2012). Probiotics-interactions with bile acids and impact on cholesterol metabolism. Applied Biochemistry and Biotechnology 168: 1880-1895.

Pereira-Fantini PM, Lapthorne S, Joyce SA, Dellios NL, Wilson G, Fouhy F, Thomas SL, Scurr M, Hill C and Gahan CG (2014). Altered FXR signalling is associated with bile acid dysmetabolism in short bowel syndrome-associated liver disease. Journal of Hepatology 61: 1115-1125.

Perry JA, Westman EL and Wright GD (2014). The antibiotic resistome: what's new? Current Opinion in Microbiology 21: 45-50.

Pinto MGV, Franz CM, Schillinger U and Holzapfel WH (2006). Lactobacillus spp. with in vitro probiotic properties from human faeces and traditional fermented products. International Journal of Food Microbiology 109: 205-214.

Qiao L, Han SI, Fang Y, Park JS, Gupta S, Gilfor D, Amorino G, Valerie K, Sealy L and Engelhardt JF (2003). Bile acid regulation of $\mathrm{C} / \mathrm{EBP} \beta$, CREB, and c-Jun function, via the extracellular signal-regulated kinase and c-Jun NH2-terminal kinase pathways, modulates the apoptotic response of hepatocytes. Molecular and Cellular Biology 23: 3052-3066.

Reinhart G, Mahan D and Cera K (1988). Effect of bile salt supplementation on tallow digestion and serum vitamin $\mathrm{E}$ concentration in weanling pigs. Nutrition Reports International 38: 563-570.

Ridlon JM, Kang D-J and Hylemon PB (2006). Bile salt biotransformations by human intestinal bacteria. Journal of Lipid Research 47: 241-259.

Roberts MS, Magnusson BM, Burczynski FJ and Weiss M (2002). Enterohepatic circulation. Clinical Pharmacokinetics 41: 751-790.

Rose P, Moore PK, Ming SH, Nam OC, Armstrong JS and Whiteman M (2005). Hydrogen sulfide protects colon cancer cells from chemopreventative agent beta-phenylethyl isothiocyanate induced apoptosis. World Journal of Gastroenterology 11: 3990.

Rossocha M, Schultz-Heienbrok R, von Moeller H, Coleman JP and Saenger W (2005). Conjugated bile acid hydrolase is a tetrameric $\mathrm{N}$-terminal thiol hydrolase with specific recognition of its cholyl but not of its tauryl product. Biochemistry 44: 5739-5748.

Russell DW (2009). Fifty years of advances in bile acid synthesis and metabolism. Journal of Lipid Research 50: S120-S125.

Schaap FG, Trauner M and Jansen PL (2014). Bile acid receptors as targets for drug development. Nature Reviews Gastroenterology \& Hepatology 11: 55-67.

Sharifi S, Dibamehr A, Lotfollahian H and Baurhoo B (2012). Effects of flavomycin and probiotic supplementation to diets containing different sources of fat on growth performance, intestinal morphology, apparent metabolizable energy, and fat digestibility in broiler chickens. Poultry Science 91: 918-927.

Sieladie DV, Zambou NF, Kaktcham PM, Cresci A and Fonteh F (2011). Probiotic properties of lactobacilli strains isolated from raw cow milk in the western highlands of Cameroon. Innovative Romanian Food Biotechnology 9: 12-28.

Smet I, Hoorde L, Woestyne M, Christiaens H and Verstraete W (1995). Significance of bile salt hydrolytic activities of lactobacilli. Journal of Applied Bacteriology 79: 292-301.

Smith K, Zeng X and Lin J (2014). Discovery of bile salt hydrolase inhibitors using an efficient high-throughput screening system. PloS ONE 9: e85344.

Sridevi N, Srivastava S, Khan BM and Prabhune AA (2009). Characterization of the smallest dimeric bile salt hydrolase from a thermophile Brevibacillus sp. Extremopbiles 13: 363-370.

Stellwag E and Hylemon P (1976). Purification and characterization of bile salt hydrolase from Bacteroides fragilis subsp. fragilis. Biocbimica et Biophysica Acta (BBA)-Ensymology 452: 165-176. 
Svensson P-A, Olsson M, Andersson-Assarsson JC, Taube M, Pereira MJ, Froguel P and Jacobson P (2013). The TGR5 gene is expressed in human subcutaneous adipose tissue and is associated with obesity, weight loss and resting metabolic rate. Biochemical and Biophysical Research Communications 433: 563-566.

Tanaka H, Hashiba H, Kok J and Mierau I (2000). Bile salt hydrolase of Bifidobacterium longum-biochemical and genetic characterization. Applied and Environmental Microbiology 66: 2502-2512.

Tremaroli V and Bäckhed F (2012). Functional interactions between the gut microbiota and host metabolism. Nature 489: 242-249.

Van Eldere J, Celis P, De Pauw G, Lesaffre E and Eyssen H (1996). Tauroconjugation of cholic acid stimulates 7 alpha-dehydroxylation by fecal bacteria. Applied and Environmental Microbiology 62: 656-661.

Van Faassen A, Bol J, van Dokkum W, Pikaar NA, Ockhuizen T and Hermus R (1987). Bile acids, neutral steroids, and bacteria in feces as affected by a mixed, a lacto-ovovegetarian, and a vegan diet. American Journal of Clinical Nutrition 46: 962-967.

Van Gaal LF, Mertens IL and Christophe E (2006). Mechanisms linking obesity with cardiovascular disease. Nature 444: 875-880.

Venneman NG and van Erpecum KJ (2010). Pathogenesis of gallstones. Gastroenterology Clinics of North America 39: 171-183.

Vlahcevic Z, Heuman D and Hylemon P (1996). Physiology and pathophysiology of enterohepatic circulation of bile acids. Hepatology: a Textbook of Liver Disease 1: 376-417.
Walker AW and Parkhill J (2013). Fighting obesity with bacteria. Science 341: 1069-1070

Wang Z, Zeng X, Mo Y, Smith K, Guo Y and Lin J (2012). Identification and characterization of a bile salt hydrolase from Lactobacillus salivarius for development of novel alternatives to antibiotic growth promoters. Applied and Environmental Microbiology 78: 8795-8802.

Watanabe M, Houten SM, Wang L, Moschetta A, Mangelsdorf DJ, Heyman RA, Moore DD and Auwerx J (2004). Bile acids lower triglyceride levels via a pathway involving FXR, SHP, and SREBP-1c. Journal of Clinical Investigation 113: 14081418.

Watanabe M, Houten SM, Mataki C, Christoffolete MA, Kim BW, Sato H, Messaddeq N, Harney JW, Ezaki O and Kodama T (2006). Bile acids induce energy expenditure by promoting intracellular thyroid hormone activation. Nature 439: 484-489.

Xu F, Guo F, Hu X-J and Lin J (2016). Crystal structure of bile salt hydrolase from Lactobacillus salivarius. Acta Crystallograpbica Section F: Structural Biology Communications 72: 376-381.

Zhang Y, Lee FY, Barrera G, Lee H, Vales C, Gonzalez FJ, Willson TM and Edwards PA (2006). Activation of the nuclear receptor FXR improves hyperglycemia and hyperlipidemia in diabetic mice. Proceedings of the National Academy of Sciences of the United States of America 103: 1006-1011. 